\title{
GRADED QUANTUM WELL STRUCTURES MADE OF DILUTED MAGNETIC SEMICONDUCTORS*
}

\author{
T. Wojtowicz, M. Kutrowski, G. KarczewsKi and J. Kossut \\ Institute of Physics, Polish Academy of Sciences \\ Al. Lotników 32/46, 02-668 Warsaw, Poland
}

In this paper we review results of studies of two types of spatially graded quantum well structures containing various layers of diluted magnetic semiconductors $\mathrm{Cd}_{1-x} \mathrm{Mn}_{x} \mathrm{Te}$ or $\mathrm{Cd}_{1-x-y} \mathrm{Mn}_{x} \mathrm{Mg}_{y} \mathrm{Te}$. The design of the structures has been recently proposed by us and suitable samples have been grown by a modified molecular beam epitaxy method. In the structures of the first type a digital profiling of the composition of the constituent material in the growth direction allowed to produce quantum wells with a specifically required shape of the confining potential (including parabolic, half-parabolic, triangular, and trapezoidal). Such samples were used for (i) determination of the conduction and valence band offsets in $\mathrm{MnTe} / \mathrm{CdTe}$ and $\mathrm{MgTe} / \mathrm{CdTe}$ systems, (ii) for the demonstration of an enhanced exciton binding in a parabolic confining potential as well as for (iii) demonstration of the possibility of "spin-splitting engineering" in diluted magnetic semiconductors quantum structures. In the second type of the structures, a precise in-plane profiling of either quantum well width or the barrier width or $n$-type doping intensity was realized. These structures were subsequently used for studies of the evolution of optical spectra with an increase in the concentration of confined two-dimensional gas of conduction electrons.

PACS numbers: 73.20.Dx, 78.55.Et

\section{Introduction}

Incorporation of paramagnetic ions (e.g., $\mathrm{Mn}$ ) in semiconductor matrices, resulting in a materials class of diluted magnetic semiconductors (DMSs), is known to modify magnetic and electronic properties of host crystals to a great extent [1-3]. Specific amplification of all spin related properties of DMSs leads to a number of interesting effects including giant Faraday rotation, giant negative magnetoresistance and magnetic field induced nonmetal-metal transition [4].

Modern growth techniques, especially molecular beam epitaxy (MBE), introduced new ways of modifying properties of II-VI DMS materials. One of these ways

* This work is supported in part by the Committee for Scientific Research (Poland) through grant 8T11B-014-11 and by the Volkswagen Foundation. 
is a possibility to grow completely new materials, such as zinc-blende MnTe [5] and zinc-blende $\mathrm{Mg}_{1-y} \mathrm{Mn}_{y} \mathrm{Te}$ [6]. Yet another possibility arises in the case of structures with reduced dimensionality, namely that of a precise spatial grading of the structure parameters in a direction either parallel [7] or perpendicular [8] to the growth axis. In this paper we will give a review of several results obtained on such graded quantum well (QW) structures recently grown by MBE method in the Institute of Physics in Warsaw. The grading was achieved either in the growth direction or in one of the directions perpendicular to the growth axis. Depending on the direction of the grading, such samples proved to be very useful in detailed investigations of several different characteristics of quantum heterostructures involving DMS materials.

\section{Quantum well structures with composition profiling in the growth direction}

Molecular beam epitaxy allows one to grow structures in which the composition of a ternary compound constituting various layers, and hence their band gaps, changes not abruptly at the interfaces, as in most typical heterostructures, but in a semi-continuous, graded fashion. The interest in such graded gap materials is motivated by their use as buffer layers in epitaxy of lattice mismatched semiconductors, as well as by their use in graded index separate confinement heterostructure lasers, in graded base transistors, and in spatially asymmetric graded heterostructure devices for current rectification and second harmonic generation, to mention only a few possible applications (for a recent review of properties and possible applications of graded gap materials see [9]). When the spatial extent of the band-gap graded region is comparable to de Broglie wavelength of the carriers then the energy of the carriers becomes quantized. The pattern of these quantized energy levels depends on the particular shape of the confining potential produced by the band gap grading. Additionally, the distances between energy levels are, contrary to the case of rectangular quantum wells, strongly sensitive to the actual value of the band gap discontinuity between the materials used to produce the graded-gap region. This makes graded QWs attractive as a tool for precise determination of band offsets.

Among different possible shapes of the confining potential, the parabolic shape attracted a special attention. The reason is that parabolic quantum wells (PQWs) not only have unique physical properties but also because they are important due to their potential application. Two such characteristic features of PQWs are their great sensitivity to an external electric field [10] and a strong enhancement of the binding energy of excitons confined in them [11]. These properties can serve as a basis for optoelectronic devices such as modulators and/or switches.

In order to produce a graded-gap quantum structure in our MBE apparatus we have used the digital growth method, originally applied by Gossard et al. [12] to fabricate PQWs from III-V materials. The method, described in detail elsewhere $[7,13]$, employs different duration of flux pulses of one of the components of the mixed crystal (e.g. $\mathrm{Mn}$, in the case of $\mathrm{Cd}_{1-x} \mathrm{Mn}_{x} \mathrm{Te}$ ). The method is, in fact, particularly appropriate in the case of $\mathrm{Cd}_{1-x} \mathrm{Mn}_{x} \mathrm{Te}$, because it involves no changes of $\mathrm{Mn}$ effusion cell temperature during growth. Due to a high value of the 
sticking coefficient of $\mathrm{Mn}$ (compared to those of $\mathrm{Cd}$ and $\mathrm{Te}$ ) one is bound to employ during the epitaxy a very small $\mathrm{Mn}$ flux which is, therefore, difficult to measure accurately making its calibration and precise control (as would be required in the analog method of growth, i.e., involving a continuous change of the molar fraction $x$ and, thus, of the Mn flux) extremely difficult.

The high precision of control of the composition, and hence the potential shape profile, achieved in our digitally-grown structures, was proved by two independent methods. The first proof comes from the transmission electron microscopy studies of specially designed trapezoidal QW structures. Kret et al. [14] analyzed the inter-atomic distance averaged in the direction perpendicular to the growth axis and concluded that the composition in the region of its nominally linear increase in reality also changes, within experimental error, linearly: The second confirmation of the precise shape modeling is provided by the magnetooptical studies of parabolic QWs and will be discussed later in this article.

Although we grew also structures with linearly varying composition, resulting in the triangular or trapezoidal shape of the confining potential [13], most of our studies was performed on structures with parabolically varying composition. A large set of parabolic and half-parabolic QW structures was produced from $\mathrm{Cd}_{1-x} \mathrm{Mn}_{x}$ Te material. A wide range of curvatures of the confining potential at its center was obtained either by changing the width (from 40 monolayers (ML) to $120 \mathrm{ML}$ ) of QWs or by varying composition of the outermost barrier material (from $x=0.14$ to $x=1)$. The structures were studied using the optical transmission method [15] (after removal of the nontransparent GaAs substrate in multiple QW structures) and by photoluminescence excitation (PLE) spectroscopy $[7,16-18]$ in magnetic fields up to $7 \mathrm{~T}$ and in the temperature range of $1.6-270 \mathrm{~K}$.

The spectra obtained in both types of experiments revealed two series of several (up to five) peaks equidistant in energy associated with "diagonal" interband excitonic transitions between harmonic oscillator levels (see further discussion of the results for $\mathrm{Cd}_{1-y} \mathrm{Mg}_{y} \mathrm{Te} \mathrm{QWs}$ ). From the analysis of the dependence of the energetic distance between the peaks in the heavy-hole diagonal series on the curvature of the potential in a large set of parabolic QWs we could determine the valence band offset value of $0.44 \pm 0.1 \mathrm{in} \mathrm{CdTe} / \mathrm{MnTe}$ system [16]. The error of the band-offset determination was further reduced by observation of "non-diagonal" transitions in both parabolic and half-parabolic QWs. Half-parabolic QWs are, in fact, preferable for the observation of the non-diagonal transitions since, due to the lack of reflection symmetry of the confining potential, all interband transitions are parity allowed. The analysis of experimental results was performed within the model assuming the same electron effective masses in $\mathrm{CdTe}$ and $\mathrm{Cd}_{1-x} \mathrm{Mn}_{x} \mathrm{Te}$ $[15,17]$ and, alternatively, using the electron mass that varies in value with the composition $x$ [18]. The result of these analyses gave the same, within the experimental uncertainty, value of the valence band offset of $0.4 \pm 0.05$.

The band-offset determination relies, of course, on the knowledge of the real shape of the confining QW potential. The equal energy spacing of the features in the spectra belonging to one series of optical transitions is already an indication that the potential we are dealing with is in fact parabolic with a considerable accuracy. A strong confirmation of the precision of the potential profiling obtained 
in our growth comes from magnetooptical studies of spin splitting of the excitonic transitions. It was recognized already long time ago that the spin splitting of the excitonic levels in a nominally rectangular CdTe QW embedded in $\mathrm{Cd}_{1-x} \mathrm{Mn}_{x} \mathrm{Te}$ barriers depends strongly on the real distribution of $\mathrm{Mn}$ ions in the interfacial regions [19]. In the case of intentionally graded quantum well structures the studies of the spin splitting provides also a very sensitive test of the actual shape of the potential. The method is based on the fact that the spin splitting in DMSs is a nonlinear function of $\mathrm{Mn}$ concentration and, therefore, the application of an external field translates into a strong perturbation of the potential profile from its zero-field shape. These deviations are most pronounced in the valence band where, in the case of a parabolic potential shape in the absence of the field, for one of the spin species (namely, for $|3 / 2,-3 / 2\rangle$ state) one can expect a "camel-back" shape of the confining potential profile after application of a magnetic field. The field-induced change of the potential shape for different spin components, which determines the field-dependence of confined energy levels to a great degree and hence the spin splitting of different excitonic transitions, depends sensitively on the actual distribution of $\mathrm{Mn}$ ions (i.e., composition profile). Therefore, very good agreement between experimentally observed spin splitting and that modeled theoretically for both parabolic [20] and half-parabolic [15] QWs provides a convincing confirmation of the precision in both potential profiling and determination of the band offset.

An interesting property of the parabolic confining potential is a strong stronger than in the case of rectangular QWs with the same width - enhancement of the exciton binding energy [11]. This can be easily understood by noting that the parabolic potential introduces a new length scale to the exciton problem $[13,15,21]$. Using this characteristic length one can estimate the binding energy of excitons within the fractional dimension approach [22]. The exciton binding energy enhancement can also be easily calculated using the variational method with a strictly two-dimensional exciton wave function and one variational parameter $[11,15]$. It is interesting to note that such simple approach gives, in the case of parabolic QWs, in contrast to rectangular QWs, quite accurate results, as was proved in comparison with corresponding results of more sophisticated theoretical models [21, 23]. The first experimental confirmation of the strong enhancement of exciton binding energies in the parabolic confining potential comes from the magnetooptical studies of half-parabolic QW [15]. In the magnetoabsorption spectra, apart from $1 s$ heavy-hole exciton feature, there were also $2 s$ and $3 s$ excited exciton states observed allowing one to determine the exciton binding energy of $22 \mathrm{meV}$ in $60 \mathrm{ML}$ wide $\mathrm{QW}$ to be compared with $11 \mathrm{meV}$ in the bulk.

In order to determine the valence band offset in $\mathrm{CdTe} / \mathrm{MgTe}$ system a set of seven parabolic QW structures made of $\mathrm{Cd}_{1-y} \mathrm{Mg}_{y} \mathrm{Te}$ was grown on (100) oriented GaAs substrates. The structures were specifically designed for the absorption experiments and therefore each of them contained either six or eight identical PQWs with $y=0$ at the center of the well. The wells were separated from each other by $260 \AA$ thick barriers. PQWs with the $\mathrm{Mg}$ content in the outermost barriers $y$ from 0.3 to 0.7 , as determined by the analysis of the post-growth X-ray diffractograms, and with nominal width of the individual well between 40 and $100 \mathrm{ML}$ 
(130 and $324 \AA$ ) were produced. Some of these structures contained additionally some percentage of $\mathrm{Mn}$ evenly distributed throughout the entire structure, the purpose of this being an easier distinguishing and unambiguous identification of transitions connected with heavy- and light-hole excitons [7].

Such PQW structures were studied by means of photoluminescence (PL) and magnetoabsorption (after removal of the nontransparent GaAs substrate) in magnetic fields up to $7 \mathrm{~T}$ and at the temperature of $T=1.8 \mathrm{~K}$. Both $\sigma^{+}$and $\sigma^{-}$ circularly polarized light with the beam perpendicular to the sample surface were used. Although the quality of optical spectra was slightly worse than in the case of a single $\mathrm{PQW}$, nevertheless, in all our samples series of peaks equidistant in energy (consisting of, typically, three peaks), associated with $\Delta n=0$ ( $n$ being the level index) excitonic transitions from the heavy-hole $\left(\mathrm{hh}_{n}\right)$ to electron $\left(\mathrm{e}_{n}\right)$ levels, were observed. Typical results are presented on the left hand side of Fig. 1, showing the data for three samples with the wells being 40,80 , and $100 \mathrm{ML}$, respectively, wide and having the $\mathrm{Mg}$ barrier concentration $y$ equal to $0.52,0.57$, and 0.71 , respectively. The identification of the peaks is given in the figure and explained in detail in the caption.

In order to determine the valence band offset we can use the infinite barrier approximation. We checked in a direct comparison with calculations suitable for finite PQWs [24] that such a simple description is very accurate in the case of our relatively deep and wide QWs. The differences between the energy values calculated for various levels electron and heavy-hole levels involved in the observed optical transitions obtained within the infinite barrier approximation and those resulting from a solution of an exact analytic equation obtained in the case a finite PQW [24] are much smaller than experimental errors.

Quantized energy levels of a carrier with the mass $m_{i}^{*}$ belonging to the $i$-th band $(i=\mathrm{e}, \mathrm{lh}, \mathrm{hh})$ bound in the infinitely high parabolic potential $V_{i}=\left(K_{i} / 2\right) z^{2}$ are given by

$$
E_{n i}=\left(n-\frac{1}{2}\right) \hbar \sqrt{\frac{K_{i}}{m_{i}^{*}}}, \quad K_{i}=Q_{i} K, \quad K=\frac{8 \Delta E_{\mathrm{g}}}{L_{z}^{2}},
$$

where $Q_{i}=\Delta E_{i} / \Delta E_{\mathrm{g}}$ with $\Delta E_{i}$ being the discontinuity of the band edges, and $\Delta E_{\mathrm{g}}$ being the energy gap discontinuity, between the materials at the center and at the distance $\pm L_{z} / 2$ from the center of the $\mathrm{QW}$, and $n=1,2,3 \ldots$ being the harmonic oscillator quantum number. Therefore, by plotting experimental values of the energy distance between two successive transitions in the heavy-hole series: $\Delta E=E_{(n+1) \mathrm{hh}}+E_{(n+1) \mathrm{e}}-E_{n \mathrm{hh}}-E_{n \mathrm{e}}$ versus square root of the total curvature $K$ at the well center one should obtain a straight line. The slope of this line is determined by the value of the valence band offset $Q_{\mathrm{hh}}\left(Q_{\mathrm{hh}}+Q_{\mathrm{e}}=1\right)$. The experiments confirm the linear dependence of $\Delta E$ vs. $\sqrt{K}$, as clearly visible on the right hand side of Fig. 1, where the data for all our samples discussed presently are collected. The curvatures of the PQWs shown in this plot were calculated taking the nominal QW widths and the experimentally determined values of $y$. From the least squares fit of a straight line to the data in Fig. 1 and using the values of the effective masses $m_{\mathrm{e}}=0.095$ for the electrons and $m_{\mathrm{hh}}=0.6$ for the heavy holes we obtained the valence band offset $Q_{\mathrm{hh}}=0.45 \pm 0.1$. This value agrees, within 

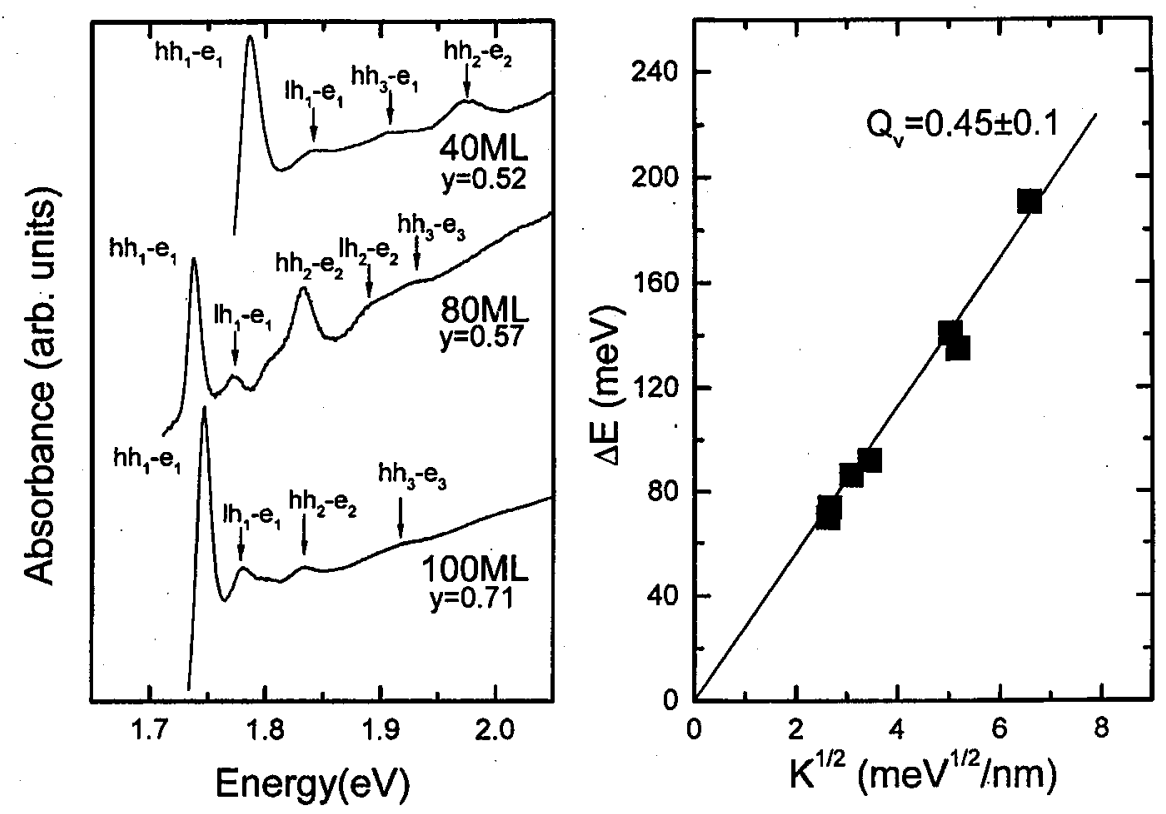

Fig. 1. Absorption spectra of three $\mathrm{Cd}_{1-y} \mathrm{Mg}_{y} \mathrm{Te} \mathrm{PQW}$ structures at $T=1.8 \mathrm{~K}$ (left panel). Each structure contained eight identical, 40, 80, or $100 \mathrm{ML}$ wide, PQWs, respectively, made of $\mathrm{Cd}_{1-y} \mathrm{Mg}_{y}$ Te with additional $\mathrm{Mn}$ evenly distributed throughout the entire width of the structures. The Mn content was similar for all the structures (about $x=0.07$ ) while the $\mathrm{Mg}$ molar fraction $y$ of the barrier is given in the figure for each structure. The identification of the lines is given by the labels $h h_{n}-e_{m}\left(l_{n}-e_{m}\right)$, where $n$ is the hh (lh) level index and $m$ is the level index in the conduction band. The right hand side of the figure shows the average energy distance between the peaks in the hh diagonal $(n=m)$ series plotted vs. square root of the curvature (at the well center) of the confining potential for all studied $\mathrm{Cd}_{1-y} \mathrm{Mg}_{y} \mathrm{Te}$ PQWs. The straight line is the least squares fit to the data and yields the valence band offset value $Q_{\mathrm{v}}=Q_{\mathrm{hh}}=0.45 \pm 0.1$ in $\mathrm{CdTe} / \mathrm{MgTe}$ system.

experimental error, with previous determinations of other authors [25]. This value is also consistent with the energy of $h_{3}-e_{1}$ non-diagonal transition observed in two $60 \mathrm{ML}$ wide $\mathrm{PQWs}$ for which the results are presented in Fig. 2. The left hand side of the figure present a comparison of the absorption spectra of these two multiple $\mathrm{Cd}_{1-y} \mathrm{Mg}_{y}$ Te PQW structures - the one containing additional evenly distributed $\operatorname{Mn}(x=0.07)$, the other without such addition. The spectrum for the structure containing no Mn was shifted up in energy in order to match the positions of the $\mathrm{hh}_{1}-\mathrm{e}_{1}$ absorption peaks. The unambiguous identification of the transitions visible in both spectra is based on their spin splitting. The right hand side of Fig. 2 presents the energy of $1 s$ excitonic features observed in magnetotransmission in the structure containing $\mathrm{Mn}$ ions as functions of the magnetic field at $T=1.8 \mathrm{~K}$. The lines represent the calculated values of the transition energy involving various 
spin-split confined states. Magnetization values in the bulk material [19] was used in these calculations. The distinction between the heavy- and light-hole excitonic transition is based on the fact that the transitions involving the light-hole levels are expected to reveal a much smaller spin splitting than those connected with the heavy holes. It is interesting to note that the spin splitting of different hh transitions shown in Fig. 2 is the same independently of the quantum numbers $m$ and $n$ of the levels involved in the transition. The cause of this fact is that in structures with an even distribution of $\mathrm{Mn}$ ions the external magnetic field does not change the potential profile, in contrast to PQWs made of $\mathrm{Cd}_{1-x} \mathrm{Mn}_{x} \mathrm{Te}$ (i.e., with $x$ varying within the samples).

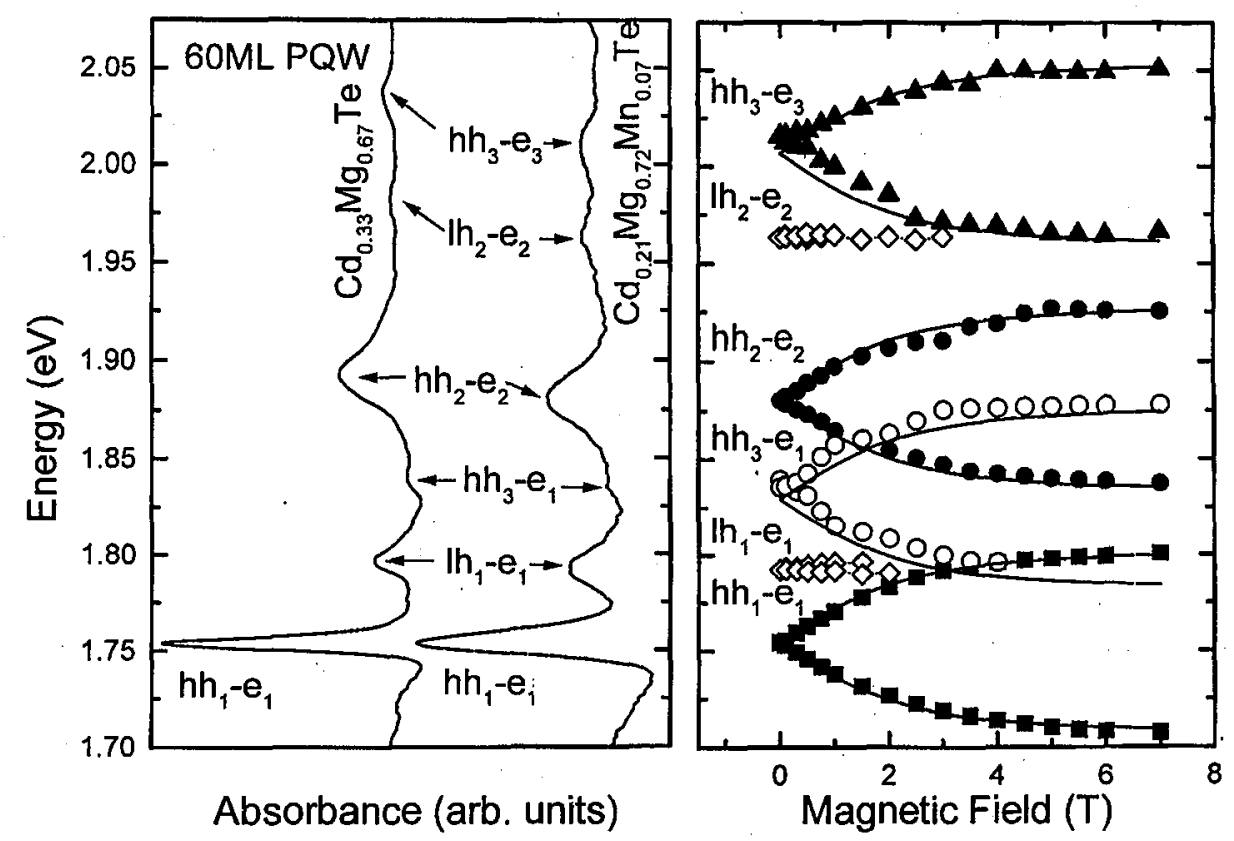

Fig. 2. Left panel: comparison of the absorption spectra of two multiple $\mathrm{Cd}_{1-y} \mathrm{Mg}_{y} \mathrm{Te}$ PQW structures, with and without additional Mn ions $(x=0.07)$ evenly distributed in the sample. Each structure contained eight PQWs with nominally the same width of $60 \mathrm{ML}$. The spectrum for the structure without $\mathrm{Mn}$ has been shifted upward in energy to match the energetic positions of $h_{1}-e_{1}$ absorption peaks. The right panel presents the energy of $1 s$ excitonic features observed in optical magnetotransmission in the structure containing $\mathrm{Mn}$ as functions of the magnetic field at $T=1.8 \mathrm{~K}$. The lines represent the calculated values of the transition energy involving various spin split confined states. The following values of the parameters were used for calculations: $m_{\mathrm{e}}=0.095, m_{\mathrm{hh}}=0.6$, $Q_{\mathrm{hh}}=0.5$ and $L_{z}=68 \mathrm{ML}$.

To finish the review of the results of studies of PQWs let us mention also a very interesting possibility of a unique "spin splitting engineering" in graded quantum structures made of quaternary $\mathrm{Cd}_{1-x-y} \mathrm{Mn}_{x} \mathrm{Mg}_{y} \mathrm{Te}$ [13]. Taking advantage of 
a very similar composition dependence of the band gap and nearly the same band offsets of $\mathrm{Cd}_{1-x} \mathrm{Mn}_{x} \mathrm{Te}$ and $\mathrm{Cd}_{1-y} \mathrm{Mg}_{y} \mathrm{Te}$, one can substitute, for a given position in the $z$-direction, a part of $\mathrm{Mg}$ atoms by the same amount of $\mathrm{Mn}$ atoms practically without affecting the potential profile in the absence of magnetic field. By properly modeling the spatial distribution of the magnetic component, one can obtain a required change of the potential profile in the presence of the magnetic field. This "spin engineering" should allow one to produce, for instance, a linear dependence of the spin splitting as a function of magnetic field.

\section{Quantum well structures with spatial in-plane profiling}

It is often of advantage, while studying many phenomena in solid state physics, to have access to samples of a material with different values of only one of the physical parameters of importance for a given phenomenon, with the remaining parameters being exactly the same. A study of the evolution of optical properties of semiconductor system with an increase in the carrier concentration and/or the reduction of the effective dimensionality of the system is a good example of such a case. Here, one would like to have at disposal a series of samples differing in carrier concentration (or well width) with values of other parameters (e.g., material composition, QW width fluctuations, etc.) being, at the same time, exactly the same in the entire series. This is, unfortunately, very difficult to achieve. A possible solution of this problem is to study a single sample in the presence of a perturbation that influences only the parameter of particular interest, say the carrier concentration $n$. For instance, in the mentioned case of studies of the evolution of optical properties of low-dimensional modulation-doped structures with an increase in two-dimensional (2D) carrier concentration three methods were used to change $n$ : (i) optical creation of the carriers [26, 27], (ii) optical depletion of the carriers [28] and (iii) the carrier removal by applying a gate voltage [29-31]. We proposed yet another and, in our opinion, quite straightforward approach, namely to study a single structure in which a smooth gradient (or a step-like profile) of values of this particular parameter (say, the electron concentration or the quantum well width) is fabricated in a controlled fashion. The structures of this kind can be grown by MBE technique by moving the main shutter (either with a constant speed or step-wise) in front of the substrate during deposition, thus producing a (continuous or step-like) profile of the width of some particular layer (e.g., the doped region or the $\mathrm{QW}$ region, in our examples). The obvious advantage of such method is that the whole structure (effectively containing an entire "set" of samples) is produced within one growth process. This makes possible to maintain the values of all other parameters at a constant value with a very high accuracy.

Let us exemplify the above idea by considering the structure with a profile of the concentration of two-dimensional electron gas (2DEG). The schematic representation of such structure is shown on the right hand side of Fig. 3. The growth has been performed on a $5 \mathrm{~cm}$ long, $5 \mathrm{~mm}$ wide (100) oriented GaAs substrate. First several layers were grown while rotating the substrate. These layers included $\mathrm{Cd}_{0.76} \mathrm{Mg}_{0.24} \mathrm{Te}$ buffer, and $81 \AA$ wide $\mathrm{QW}$ of $\mathrm{Cd}_{0.99} \mathrm{Mn}_{0.01} \mathrm{Te}$ followed by a $100 \AA$ wide spacer. Then, the rotation of the substrate was stopped and the growth of iodine doped $\mathrm{Cd}_{0.76} \mathrm{Mg}_{0.24} \mathrm{Te}$ region was started with the main shutter 


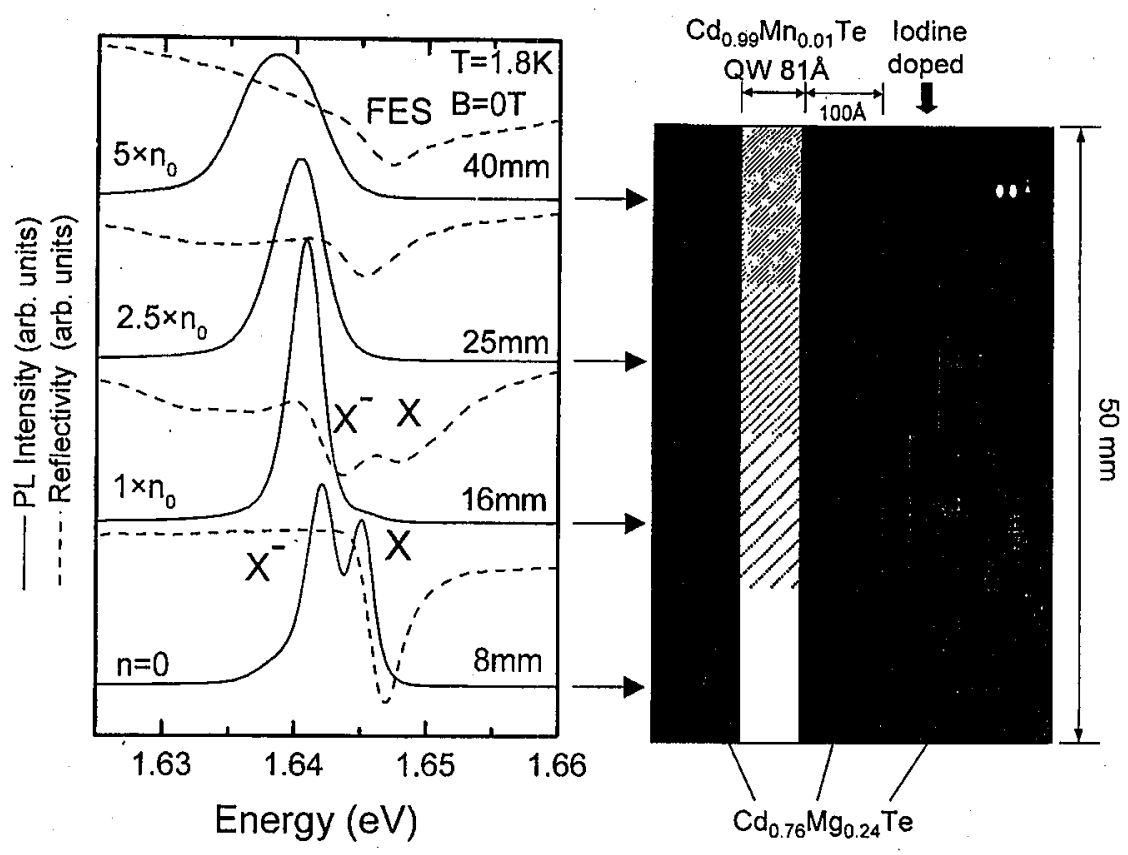

Fig. 3. Photoluminescence (solid lines) and reflectivity (dashed lines) of four regions of wedge-modulation doped structure with iodine donors introduced $100 \AA$ away from the $81 \AA$ wide $\mathrm{Cd}_{0.99} \mathrm{Mn}_{0.01} \mathrm{Te} \mathrm{QW}$. Details of the structure design are shown by the scheme on the right hand side. Different shading of the $Q W$ region represents varying 2DEG concentration.

being moved at the same time in four $11 \mathrm{~mm}$ long steps whose edges were perpendicular to the long axis of the substrate. This resulted in different thicknesses of the doped region $(0,19,49$, and $100 \AA$, respectively) and, thus, in different numbers of donors introduced to different parts of the structure. After completion of the doping procedure the remaining $500 \AA$ undoped barrier was grown while rotating the substrate again. As a result of such growth procedure we obtained the modulation-doped structure having two-dimensional electron gas with the density varying from nominally 0 at the one end of the sample to about $5 \times 10^{11} \mathrm{~cm}^{-2}$ at the other.

The photoluminescence and reflectivity spectra collected from different parts of this particular structure are presented on the left hand side of Fig. 3. Both show an evolution with the increase in 2DEG concentration. The gradual changes in the spectra are quite pronounced and easy to follow. The reflectivity spectrum of the undoped part reveals one clear feature connected with creation of neutral excitons. In the PL spectrum two peaks are seen: the one at higher energy is due to excitons localized by the well width fluctuations and the lower energetic peak is due to recombination of negatively charged excitons $X^{-}$[32] (for a review of $X^{-}$complexes see Ref. [33]). With an increase in $n$ (spectra from the region with $n=n_{0}$ ) 
a strong $X^{-}$line appears in the reflectivity and the $X^{-}$emission dominates the corresponding PL spectrum. At the highest concentration $\left(5 \times n_{0} \approx 5 \times 10^{11} \mathrm{~cm}^{-2}\right.$ - determined from the value of the filling factor $\nu=3$ observed at $B \approx 6.5 \mathrm{~T}$ in our magnetooptical studies) a broad bump-like structure due to the band-to-band recombination in the PL and a broad structure in the reflectivity, related to transitions between the valence band and the unoccupied conduction band states above the Fermi level enhanced by many-body correlation (Fermi edge singularity FES) are observed. The energy difference between the features observed in PL and reflectivity represents the Moss-Burstein shift. It is also important to note that, as clearly visible in Fig. 3, the energy of the main feature in the reflectivity remains nearly exactly (within $\approx 3 \mathrm{meV}-$ as compared to neutral exciton binding energy of about $20 \mathrm{meV}$ ) at the same position corresponding to the unscreened exciton energy, regardless of the concentration of the electrons. This fact, predicted theoretically by Hawrylak [34], is now very convinçingly confirmed by our experiments since they were performed on one sample.

Magnetooptical studies of this sample have been also performed [8]. Apart from the giant spin splitting of the exciton, which is characteristic of DMSs, we observed also two combined exciton cyclotron resonances $\left(\mathrm{ExCR}_{1}\right.$ and $\left.\mathrm{ExCR}_{2}\right)$ [26] as well as optical transitions between spin-polarized Landau levels. The circular dichroism of the $X^{-}$line consistent with the giant spin splitting (corresponding to a positive $g$-factor) was observed [8] - see the discussion of results presented in Fig. 6 below. Unfortunately, since the same degree of circular dichroism is expected for donor bound excitons $\left(D^{0} X\right)$, this property alone cannot be used to unambiguously identify $X^{-}$. Therefore, three additional methods were employed to make sure that in iodine modulation doped $\mathrm{CdTe} / \mathrm{Cd}_{1-y} \mathrm{Mg}_{y} \mathrm{Te}$ and $\mathrm{Cd}_{1-x} \mathrm{Mn}_{x} \mathrm{Te} / \mathrm{Cd}_{1-y} \mathrm{Mg}_{y} \mathrm{Te}$ structures we, in reality, observe negatively charged excitons and not just $D^{0} X$.

First $\mathrm{Hu}$ et al. [35] used microwave induced changes of the photoluminescence intensity as a tool to provide a clue to the trion origin of the low energy peak observed in the PL. These measurements were performed in samples containing $81 \AA$ wide QWs separated by $\mathrm{Cd}_{1-y} \mathrm{Mg}_{y}$ Te barriers from either (i) a suitably designed superlattice (see [35]) or (ii) a doped region in a modulation doped structure. In the sample of the first type, electrons and holes were created in the superlattice region by illumination. Because of very different probabilities of tunneling of electrons and holes to the $\mathrm{QW}$ region it was possible to produce excessive 2DEG inside the QW [26]. In order to optimize the width of the tunneling barrier, so that the maximum excessive 2DEG concentration could be obtained, a special structure having a step-like profile of the barrier width was produced. It was found that a $200 \AA$ wide barrier was the best and, therefore, the sample with this barrier width was used for further experiments. It was observed that upon irradiating the sample with microwaves the intensity of the PL line due to exciton recombination increases strongly and, at the same time, the intensity of the PL line attributed to $X^{-}$recombination strongly decreases. This behavior can be explained as caused by heating of electrons by microwave power leading to a reduction of the probability of formation of $X^{-}$and, hence, to a drop of intensity of the $X^{-}$PL peak. Because $X^{-}$PL occurs at the expense of $X$-luminescence, the reduction of $X^{-}$-luminescence intensity leads to an increase in the $X$ peak. Such 
a strong heating effect is not expected to occur in the case of carriers localized on donors, therefore, the experiments of $\mathrm{Hu}$ et al. (a) prove that free carriers are in fact present inside the $\mathrm{QW}$ and (b) corroborate the interpretation ascribing the line located below the excitonic feature in the luminescence to $X^{-}$complexes.

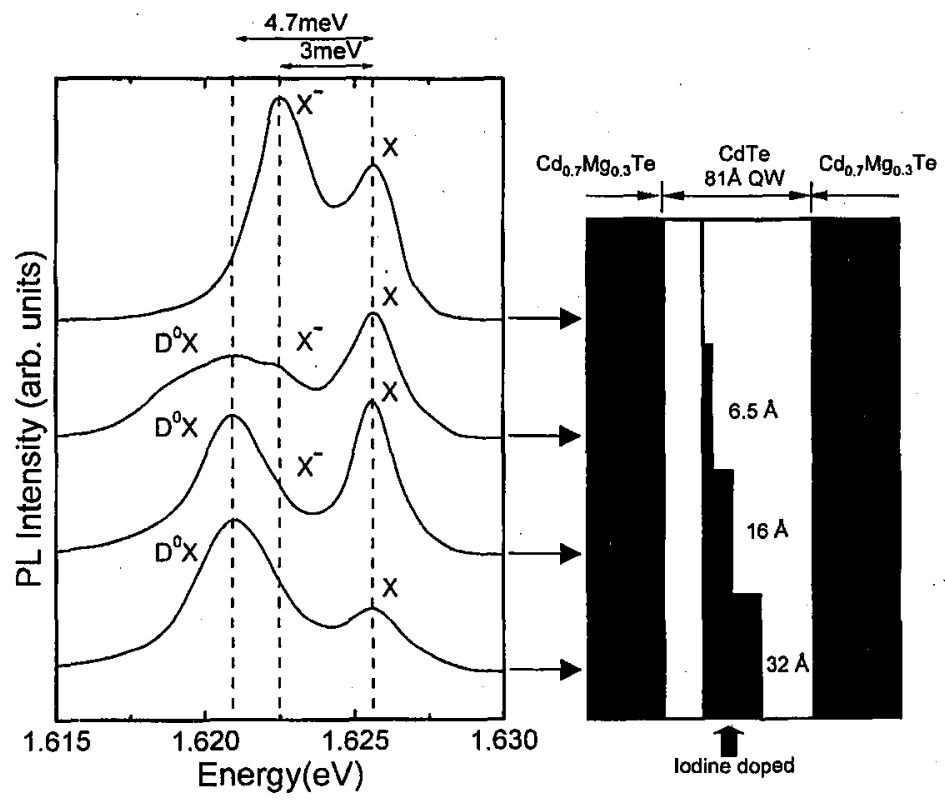

Fig. 4. Intensity of photoluminescence excited in four different regions of structure "wedge" doped inside the $81 \AA$ wide CdTe QW. Details of the structure design are shown by the scheme on the right hand side. Peaks labeled $D^{\circ} X, X^{-}$, and $X$ are connected with the recombination of donor bound excitons, negatively charged excitons and excitons localized by potential fluctuations, respectively.

Another strong support comes from the PL studies of the structure with a step-like intensity of $n$-type doping inside the QW region. The scheme of the sample is given on the right hand side of Fig. 4 . The left hand side of the same figure shows the photoluminescence collected from different parts of this structure. The spectra from the undoped and the most heavily doped parts are shifted in energy so that the positions of neutral exciton peaks coincide in the entire sample (the shift compensates a small, natural gradient of the QW width connected with inhomogeneity of flux in the MBE apparatus). PL spectra from all various regions exhibit two peaks. The peak at a higher energy is due to the recombination of neutral excitons localized by potential fluctuations. We attribute the lower energy peak in the nominally undoped region to the recombination of negatively charged excitons. Their existence is connected with a nonvanishing concentration of electrons originating from the residual donors present in the structure. The distance in energy between the two peaks, equal approximately to $3 \mathrm{meV}$, corresponds to the distance between the $X$ and $X^{-}$peaks observed in PL and in reflectivity in 
weakly modulation doped structures with the same QW width. With an increase in the width of the doped region, and hence with an increase in the number of donors present inside the QW, another peak, located at $4.7 \mathrm{meV}$ below $X$, starts to appear and gains in intensity. The larger value of the binding energy as well as the correlation of the intensity with the number of donors point to recombination of excitons localized by neutral donors, $D^{0} X$, as the origin of this peak.

Finally, we have produced a series of "wedge modulation doped" samples (having either $\mathrm{CdTe}$ or $\mathrm{Cd}_{1-x} \mathrm{Mn}_{x} \mathrm{Te}$ as QWs) similar in design to the sample schematically shown in Fig. 3 but with a smaller range of 2DEG concentration variation (with the width of the doped region $0,6.5,16$, and $32 \AA$, respectively). In these structures we observed in PL, PLE, reflectivity, and absorption a progressive increase in the intensity of the $X^{-}$line with respect to the $X$ line and a broadening of the latter (cf. Fig. 6) with 2DEG concentration. This again indicates that negatively charged excitons are formed in our samples.

Summing up this part, the microwave experiments, the correlation of the intensity of $X^{-}$line with the increase in the electron concentration, the increase in $D^{0} X$ line with the increase in the number of donors inside $\mathrm{QW}$, the observed difference between $D^{0} X$ and $X^{-}$binding energies, and, finally, the fact that $X^{-}$ line is very intense (sometimes even stronger than the $X$ line) in our transmission, reflectivity, and PLE experiments, all provide sufficient grounds for clear distinction between $D^{0} X$ and $X^{-}$lines.

Having unambiguously identified the negatively charged excitons (or trions) we turned to studies of their binding energy. Surprisingly, extended studies of this basic characteristics of $X^{-}$was performed neither in the case of III-V nor II-VI semiconductor structures. Samples with only a very limited number of different well widths were measured so far. For our studies two series of different types of "wedge" structures were prepared. The first series consisted of samples having different widths of the QW in different structures but the same kind of step-like profile of doping $(0,2,5$, and $10 \mathrm{ML}$ wide steps). The second series contained structures with a step-like profile of the width of the $\mathrm{QW}$ and the same doping level [8]. The results for some of these samples studied so far are presented in Fig. 5 . The points represent our experimental data obtained from the energy separation between $X$ and $X^{-}$lines observed in PL (rectangles) and in reflectivity or PLE (circles). We found that for a given QW width the binding energy, determined in this way, depends on the electron concentration. The dependence is weak in the limit of small concentration of 2DEG, therefore we plotted in Fig. 5 only the results for the parts of the samples with the weakest doping. The literature data obtained from the studies of $\mathrm{CdTe} / \mathrm{Cd}_{1-z-y} \mathrm{Zn}_{z} \mathrm{Mg}_{y} \mathrm{Te}$ modulation doped structures made by MBE in Grenoble [32, 31, 36] are also plotted in Fig. 5 for comparison. A strong increase in $X^{-}$binding energy from $1.25 \mathrm{meV}$ in $500 \AA$ wide QW to the $4.4 \mathrm{meV}$ in $38 \AA$ wide $\mathrm{QW}$ is clearly visible in this figure. The increase, as predicted theoretically [37], is much stronger than the one observed for neutral excitons. This fact can be intuitively understood on the basis of a larger spatial extent of the wave function of the second electron forming $X^{-}$as compared to the extension of the wave function of the electron in neutral exciton. Therefore, there is a stronger influence of the confinement imposed by the $\mathrm{QW}$ on 


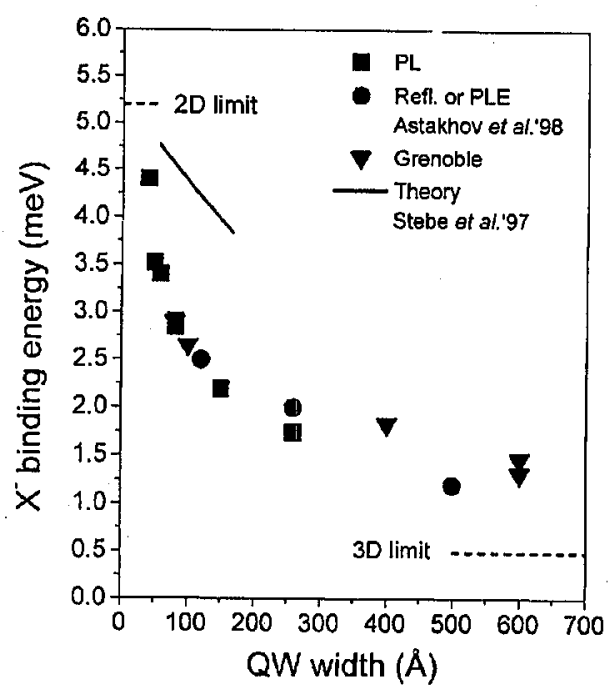

Fig. 5. Binding energy of negatively charged excitons defined as the energy separation between the peaks connected to $X$ and $X^{-}$. Points represent the experimental data obtained by PL (rectangles) and PLE or reflectivity (circles) studies of our "wedge" $\mathrm{CdTe} / \mathrm{Cd}_{0.7} \mathrm{Mg}_{0.3} \mathrm{Te}$ structures. The triangles show the results of measurements in $\mathrm{CdTe} / \mathrm{Cd}_{1-x-y} \mathrm{Zn}_{x} \mathrm{Mg}_{y} \mathrm{Te}$ samples grown in Grenoble [32, 31, 36]. The solid line represents the rescaled theoretical calculation of Stébé et al. [38].

the second electron. The only published, so far, theoretical calculation of the trion binding energy as a function of the well width is done by Stébé et al. [38]. Although it originally concerned $\mathrm{GaAs} / \mathrm{GaAlAs}$, it can be easily rescaled to our situation. This is because the Hamiltonian describing the trions, if expressed in natural units of energy and length (effective Rydberg and the Bohr radius), depends only on the mass ratio $\sigma=m_{\mathrm{e}} / m_{\mathrm{h}}$ and on the band offsets. The calculation of Ref. [38] was performed for $Q_{\mathrm{v}}=0.43$ and $\sigma=0.196$. Fortunately, the band offset in $\mathrm{CdTe} / \mathrm{MgTe}$ system, as we determined from the studies of PQWs, is nearly the same. It is also not unreasonable, in the case of $\mathrm{CdTe} / \mathrm{CdMgTe}$ structures, to take $\sigma=0.2$ as shown by Yakovlev et al. [26] from studies of neutral excitons in a magnetic field. Thus, it is possible to rescale the results of Stébé to the case of $\mathrm{CdTe} / \mathrm{Cd}_{0.7} \mathrm{Mg}_{0.3} \mathrm{Te}$ by simply changing the energy and the length scales by using appropriate values of the exciton Rydberg and the Bohr radius in CdTe. The result of such rescaling is shown in Fig. 5 by the solid line. Noticeable disagreements between our experiments and the theory call for a refinement of the latter. We hope that our experimental results will, in fact, stimulate further development of the theory of the binding energy of trions.

Incorporation of $\mathrm{Mn}$ ions in the $\mathrm{QW}$ region in modulation doped structures offers a possibility of tailoring the sign and the magnitude of the effective $g_{\mathrm{eff}}^{*}$-factor of two-dimensional electron gas. In DMS $g_{\mathrm{eff}}^{*}$ is described by the sum of the intrinsic $g_{\mathrm{in}}^{*}$ factor and the contribution $g_{\text {exch }}^{*}$ due to $s-d$ exchange interaction 


$$
g_{\mathrm{eff}}^{*}=g_{\mathrm{in}}^{*}+g_{\mathrm{exch}}^{*}=g_{\mathrm{in}}^{*}+\alpha M(B, T) /\left(g_{\mathrm{Mn}} \mu_{\mathrm{B}}^{2} B\right) .
$$

The exchange part $g_{\text {exch }}^{*}$ is proportional to the exchange constant $\alpha$ and the magnetization $M$ which, in turn, can be modeled by a modified Brillouin function [19]. Thus, $g_{\text {exch }}^{*}$ depends on the Mn molar fraction $x$, the temperature $T$ and magnetic field $B$. In our case, a realistically possible to achieve variation of $g_{\mathrm{eff}}^{*}$ spans at low fields $g_{\mathrm{eff}}^{*}=-1.6$ in CdTe and $g_{\mathrm{eff}}^{*} \approx+100$ in $\mathrm{Cd}_{0.98} \mathrm{Mn}_{0.02} \mathrm{Te}$ at helium temperatures (here we limit ourselves to $x \leq 0.02$ simply in order to avoid degradation of optical quality of the ternary material forming the QWs). For these reasons, in $\mathrm{Cd}_{1-x} \mathrm{Mn}_{x} \mathrm{Te} \mathrm{QW}$ structures that additionally possess $2 \mathrm{DEG}$ concentration grading within the QW plane one can investigate with a great precision what is the role of the spin degree of freedom in modifications of optical properties due to the presence of $2 D E G$. We performed such studies in a series of structures having different composition of Mn (from $x=0$ up to $x=0.01$ ), and thus, different $g_{\mathrm{eff}}^{*}$. As mentioned, $g_{\text {eff }}^{*}$ can be additionally modified by changing the temperature and the magnetic field. Therefore, the magneto-reflectivity for both $\sigma^{+}$and $\sigma^{-}$circular

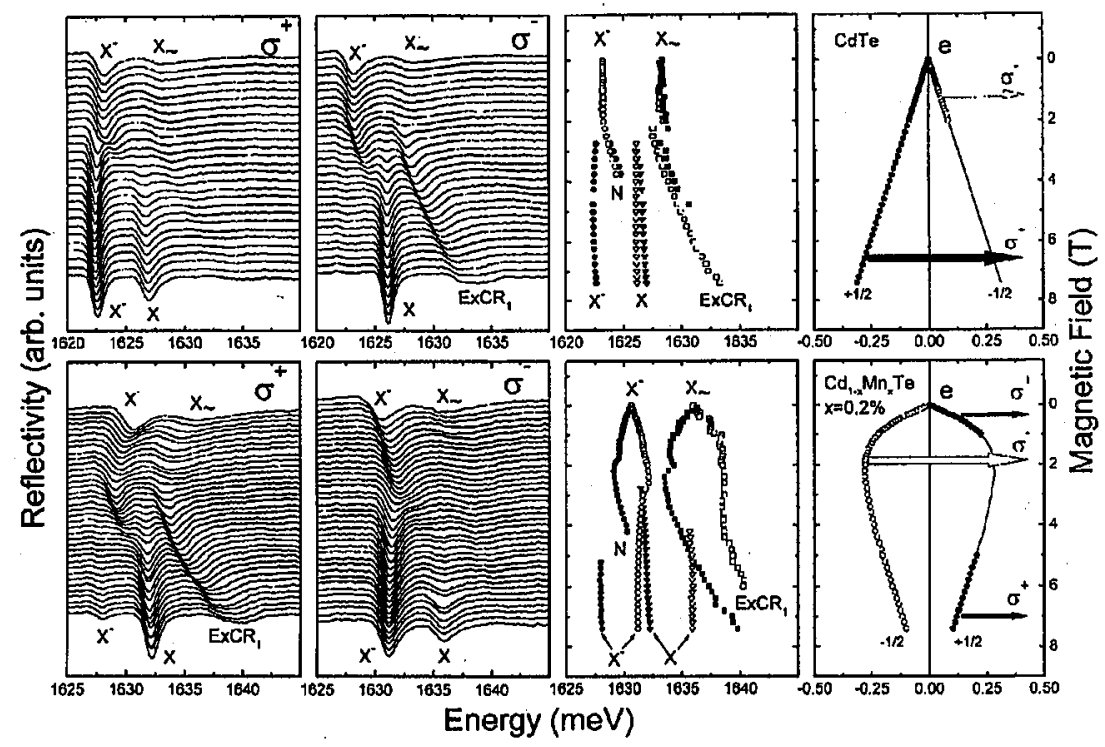

Fig. 6. Comparison of the results of the magnetoreflectivity studies of two modulation doped structures containing CdTe (upper row of panels) and $\mathrm{Cd}_{0.998} \mathrm{Mn}_{0.002} \mathrm{Te}$ (lower row) QW with similar concentration of 2DEG. The spectra in panels forming two left columns were collected for different magnetic fields at $1.8 \mathrm{~K}$ in both $\sigma^{+}$and $\sigma^{-}$circular polarizations. The magnetic field increases from 0 to $7.5 \mathrm{~T}$ from the top to the bottom spectrum in each panel with the step of $0.25 \mathrm{~T}$ for $\mathrm{CdTe}(0.2 \mathrm{~T}$ in the case of $\mathrm{Cd}_{0.998} \mathrm{Mn}_{0.002} \mathrm{Te} \mathrm{QW}$ ) between two consecutive spectra. The panel forming two right columns shows positions of the observed peaks vs. magnetic field and (the rightmost column) experimentally determined spin splitting of the electron levels which are the initial states for the creation of trions. 
polarizations was measured in magnetic fields up to $7.5 \mathrm{~T}$ and in a temperature range from 1.6 up to $70 \mathrm{~K}$. Our studies demonstrate that the sign and the magnitude of the circular dichroism of both $X^{-}$and the combined exciton-cyclotron resonances $\left(\mathrm{ExCR}_{n}\right)$ lines are predetermined by the sign and the magnitude of the effective $g^{*}$ and that they can be tuned by tuning $g^{*}$.

An example of the results of these studies is presented in Fig. 6 which shows the comparison of the magnetoreflectivity data obtained on two "wedge modulation doped" structures containing either CdTe QW (upper row of panels) or $\mathrm{Cd}_{0.998} \mathrm{Mn}_{0.002} \mathrm{Te} \mathrm{QW}$ (lower row of panels). Both structures had the same width of the QW $(81 \AA)$ and the same width of the spacer $(100 \AA)$. The data were collected from the regions of the samples having a very similar, and relatively high - of the order of $1 \times 10^{11} \mathrm{~cm}^{-2}$ - concentration of 2DEG. The series of spectra presented in panels forming two left columns were measured for different magnetic fields from 0 (top spectrum in each panel) to $7.5 \mathrm{~T}$ (bottom spectrum in each panel) at $1.8 \mathrm{~K}$. The panels forming two right columns show positions of the observed peaks vs. the magnetic field and (the rightmost panels) the spin splitting of the electron levels. The electron spin splitting was determined, in the case of CdTe QW, from the optically detected magnetic resonance (ODMR) experiment [35] and, in the case of $\mathrm{Cd}_{0.998} \mathrm{Mn}_{0.002} \mathrm{Te} \mathrm{QW}$, from the exciton splitting observed in the undoped part of the sample taking the ratio of $p-d$ and $s-d$ exchange constants $\beta / \alpha=4$, i.e., as in the bulk material [2] (both the valence and the conduction band $g$-factors contribute, of course, to the resultant spin splitting of the exciton). The correctness of the latter procedure was checked by the comparison of the above results with the spin splitting of electrons directly measured in spin-flip Raman scattering experiments [39].

The reflectivity spectra presented in two left columns reveal two features at zero magnetic field. The one at a lower energy is connected to a creation of negatively charged exciton complex and marked $X^{-}$. The feature at a higher energy, marked $X_{\sim}$, is connected to creation of a "neutral" exciton that is strongly modified by the presence of $2 \mathrm{DEG}$. This feature is considerably broadened, especially on the high energy side. A similar broadening was observed before both in the reflectivity and the PLE spectra observed in "wedge doped" $\mathrm{CdTe} / \mathrm{Cd}_{1-y} \mathrm{Mg}_{y} \mathrm{Te}$ structures produced also by us [40]. The broadening is related to a strong scattering of excitons by electrons from the continuum (at zero magnetic field) of eigenenergies.

Upon the application of a magnetic field the spectrum observed in CdTe QW changes (upper row in Fig. 6): first, the $X^{-}$line becomes stronger in $\sigma^{+}$ polarization while it disappears in $\sigma^{-}$polarization. This behavior reflects the fact that the ground state of the trion is a singlet. Electrons, being initial states for the creation of trions and having negative $g_{\text {eff }}^{*}$, occupy at a low temperature and in a high field, only $|+1 / 2\rangle$ state (schematically shown as dots in the upper panel in the fourth column of Fig. 6), therefore, the creation of $X^{-}$is possible by $\sigma^{+}$ excitation.

Secondly, since in the presence of the field the energies of the conduction electrons becorne quantized, the high energy line becomes sharper with the field increasing, finally to resemble closely the exciton line observable in samples without 
2DEG. At the same time, in $\sigma^{-}$polarization a new line appears, marked ExCR ${ }_{1}$. It is connected to the combined exciton cyclotron process, i.e., with the creation of excitons with a simultaneous excitation of an electron from the first Landau level $\left(L_{L}\right)$ to the second Landau level $\left(\mathrm{LL}_{1}\right)$ [26]. In a more strongly doped part of the same sample than that presented in Fig. 6 even $\mathrm{ExCR}_{2}$ lines - with an electron excited from $\mathrm{LL}_{0}$ to $\mathrm{LL}_{2}$ - were observed.

The polarization behavior of the magnetoreflectivity of the sample containing ternary $\mathrm{Cd}_{0.998} \mathrm{Mn}_{0.002} \mathrm{Te} \mathrm{QW}$ is markedly different from that containing binary CdTe QW. First of all, the spin splitting of the excitonic transitions has a much larger magnitude than that in $\mathrm{CdTe}$ (this is due to the giant spin splitting of both the conduction and valence band edges in DMSs). Secondly, polarization properties of all features observed in reflectivity is just reversed. Now, it is the $X^{-}$line that disappears in $\sigma^{+}$and the $\mathrm{ExCR}_{1}$ line is strong in this polarization. Additionally, the $X^{-}$line reappears in $\sigma^{+}$at magnetic fields of about $5.5 \mathrm{~T}$. Such reversed polarization behavior can be in fact expected for this structure containing QW made of DMS. In fact, Mn molar fraction in QW material of this sample was chosen such that the electron spin splitting showed a nonmonotonic dependence on the external magnetic field in the range from 0 to $7.5 \mathrm{~T}$ (see the lower right panel in Fig. 6). At weak magnetic fields the electron spin splitting is dominated by a positive contribution due to the exchange $\left(g_{\mathrm{eff}}^{*}(B \rightarrow 0) \approx g_{\mathrm{exch}}^{*}=+10\right)$. With the magnetic field increasing the exchange contribution saturates (reflecting the saturation of the magnetization). Starting from this moment the field dependence of the splitting is determined by the negative intrinsic $g_{\mathrm{in}}^{*}$ factor, and the splitting hence decreases. Therefore, with the increase in the magnetic field, the preexisting electrons occupy, at a low temperature, preferentially $|-1 / 2\rangle$ spin sublevels (see the lower panel in the rightmost column of Fig. 6). The population of $|+1 / 2\rangle$ decreases at first, and therefore the intensity of the $X^{-}$line decreases with $B$ in $\sigma^{+}$polarization until it disappears completely. In still higher magnetic fields when the spin splitting starts to decrease - the $|+1 / 2\rangle$ state becomes to be populated again. This is the moment when the $X^{-}$in $\sigma^{+}$polarization reappears and gains in the intensity.

It can be expected that for still higher magnetic fields the $|-1 / 2\rangle$ and $|+1 / 2\rangle$ states will finally cross. Then the $|+1 / 2\rangle$ state becomes the ground state. In terms of their population $|+1 / 2\rangle$ becomes then occupied with electrons while $|-1 / 2\rangle$ becomes empty. Therefore, one expects that at sufficiently high magnetic fields, when $g_{\text {eff }}^{*}$ becomes negative, the behavior will be similar to that in the case of CdTe QW. Specifically, the $X^{-}$line should become more intense in $\sigma^{+}$polarization while it should be losing intensity to disappear ultimately in $\sigma^{-}$. Such behavior was actually observed by us [41]. Elements of similar behavior was also observed by Cox et al. [33], however, the electron concentration in their sample being much higher than our upper range limit of 2DEG concentrations, the $X^{-}$line could not be observed by them at $B=0$ but only above $4.5 \mathrm{~T}$. Therefore, the full complexity of the changes of polarization behavior were not observed in Ref. [33].

Let us finally note that, in addition to $X, X^{-}$, and $\mathrm{ExCR}_{n}$ lines, a new feature, marked by $N$ in Fig. 6, was observed in our samples containing CdTe (where it was more pronounced in $\sigma^{-}$polarization) as well as $\mathrm{Cd}_{0.998} \mathrm{Mn}_{0.002} \mathrm{Te}$ 
QWs (where it is stronger in $\sigma^{+}$). Its slope vs. the field corresponds roughly to $\hbar \omega_{\mathrm{c}}^{\mathrm{e}} / 2$ (electron cyclotron energy). Although the origin of this line is not yet fully understood, it, was suggested by Suris and Yakovlev [42] that it can be due to a creation of the trion $X^{-}$with a simultaneous excitation of one electron from $\mathrm{LL}_{0}$ to $L_{1}$. Such an excitation would be an analog of the ExCR process, however, now with two electrons and a photon being the initial particles, instead of one electron and a photon being initial state for the ExCR. More work, both experimental and theoretical, is of course necessary to confirm this interpretation.

\section{Conclusions}

In this paper we tried to give a short review of magnetooptical studies of two types of novel DMS quantum well structures with a grading of some parameters in the direction either parallel (resulting in $\mathrm{QW}$ having a different shape of potential) or perpendicular (resulting in "wedge-doped", "wedge-well", or "wedge-barrier" QW structures) to the growth axis. This review, because of its space limitations, is far from being complete. The reader is further referred to several papers, mentioned in this review, presenting in more detail results of various types of measurements taken recently on our samples. We hope that the unique structures developed in our laboratory, especially those with spatial in-plane profiling of the barrier width and the doping intensity, will be useful in future studies of other important problems of solid state physics, including the studies of the influence of spin degree of freedom on the band gap renormalization and studies of spin dependent exciton and carrier tunneling.

\section{Acknowledgments}

The present review is based in parts on the collaboration with G. Cywinski, E. Dynowska, M. Godlewski, E. Janik, F. Kyrychenko, K. Kopalko, P. Kossacki, S. Kret, D.R. Yakovlev, W. Ossau, R. Fiederling, C.Y. Hu, A. Keller, G. Landwehr, G. Astakhov, V.P. Kochereshko, R.A. Suris, F.J.T. Garcinuno, and M. Potemski.

\section{References}

[1] Diluted Magnetic Semiconductors, Eds. J.K. Furdyna, J. Kossut, in series Semiconductors and Semimetals, Vol. 25, Academic Press, Boston 1987.

[2] J. Kossut, W. Dobrowolski, in: Handbook of Magnetic Materials, Ed. K.H.J. Buschow, Vol. 7, Elsevier North-Holland, Amsterdam 1993, Ch. 4, p. 231.

[3] T. Dietl, in: Materials, Properties and Preparation, Ed. S. Mahajan, in series: Handbook on Semiconductors, Ed. T.S. Moss, Vol. 3, Elsevier, Amsterdam 1994, Ch. 17.

[4] T. Wojtowicz, T. Dietl, M. Sawicki, W. Plesiewicz, J. Jaroszyński, Phys. Rev. Lett. 56, 2419 (1986).

[5] E. Janik, E. Dynowska, E. Bąk-Misiuk, M. Leszczyński, W. Szuszkiewicz, T. Wojtowicz, G. Karczewski, A.K. Zakrzewski, J. Kossut, Thin Solid Films 276, 74 (1995).

[6] E. Janik, E. Dynowska, E. Bąk-Misiuk, T. Wojtowicz, G. Karczewski, J. Kossut, A. Stachow-Wójcik, A. Twardowski, W. Mac, K. Ando, J. Cryst. Growth 184/185, 976 (1998). 
[7] T. Wojtowicz, M. Kutrowski, M. Surma, P. Kossacki, Nguyen The Khoi, K. Kopalko, G. Karczewski, J. Kossut, M. Godlewski, Appl. Phys. Lett. 68, 3326 (1996).

[8] T. Wojtowicz, M. Kutrowski, G. Karczewski, J. Kossut, Appl. Phys. Lett., accepted.

[9] A.C. Gossard, M. Sundaram, P.F. Hopkins, in: Epitaxial Microstructures, Ed. A.C. Gossard, in series: Semiconductors and Semimetals, Vol. 40, Academic Press, Boston 1994, p. 153.

[10] W.P. Shen, M.L. Rustgi, J. Appl. Phys. 74, 4006 (1993).

[11] Y. Chu-Liang, Y. Quing, Phys. Rev. B 37, 1364 (1988).

[12] A.C. Gossard, R.C. Miller, W. Wiegmann, Surf. Sci. 174, 131 (1986).

[13] T. Wojtowicz, G. Karczewski, J. Kossut, Thin Solid Films 306, 271 (1997).

[14] S. Kret, C. Delamarre, J.Y. Laval, A. Dubon, to be published.

[15] T. Wojtowicz, M. Kutrowski, G. Cywiński, G. Karczewski, E. Janik, E. Dynowska, J. Kossut, R. Fiederling, A. Pfeuffer-Jeschke, W. Ossau, J. Cryst. Growth 184/185, 936 (1998).

[16] T. Wojtowicz, M. Kutrowski, G. Cywiński, E. Dynowska, G. Karczewski, J. Kossut, R. Fiederling, G. Mackh, U. Zehnder, W. Ossau, Acta Phys. Pol. A 90, 977 (1996).

[17] M. Kutrowski, T. Wojtowicz, G. Cywiński, G. Karczewski, E. Janik, E. Dynowska, J. Kossut, P. Kossacki, R. Fiederling, A. Pfeuffer-Jeschke, W. Ossau, Acta Phys. Pol. A 92, 887 (1997).

[18] W. Ossau, R. Fiederling, B. König, T. Wojtowicz, M. Kutrowski, G. Karczewski, J. Kossut, Phys. Low-Dim. Struct. 11/12, 89 (1997).

[19] J.A. Gaj, W. Grieshaber, C. Bodin-Deshayes, J. Cibert, G. Feuillet, Y. Merle d'Aubigné, A. Wasiela, Phys. Rev. B 50, 5512 (1994).

[20] W. Ossau, R. Fiederling, B. König, T. Wojtowicz, M. Kutrowski, G. Karczewski, J. Kossut, Physica B, in print.

[21] F. Kyrychenko, J. Kossut, Acta Phys. Pol. A 94 (1998), Proc. of this Conf. (Part II); Sem. Sci. Technol., in print.

[22] H. Mathieu, P. Lefebvre, P. Christol, Phys. Rev. B 46, 4092 (1992).

[23] T. Rusin, to be published.

[24] W.-P. Yuen, Phys. Rev. B 48, 17316 (1993).

[25] B. Kuhn-Heinrich, W. Ossau, H. Heinke, F. Fisher, T. Litz, A. Waag, G. Landwehr, Appl. Phys. Lett. 63, 2932 (1993).

[26] D.R. Yakovlev, V.P. Kochereshko, R.A. Suris, H. Schenk, W. Ossau, A. Waag, G. Landwehr, P.C.M. Christianen, J.C. Maan, Phys. Rev. Lett. 79, 3974 (1997).

[27] R.B. Miller, T. Baron, R.T. Cox, K. Saminadayar, J. Cryst. Growth 184/185, 822 (1998).

[28] B.M. Ashkinadze, A. Nazimov, E. Cohen, A. Ron, L.N. Pfeiffer, Phys. Status Solidi A 164, 523 (1997).

[29] A.J. Shields, M. Papper, D.A. Ritchie, M.Y. Simmons, G.A.C. Jones, Phys. Rev. $B$ 51, 18049 (1995).

[30] G. Finkelstein, H. Shtrikman, I. Bar-Joseph, Phys. Rev. B 53, R1709 (1996).

[31] S. Lovisa, R.T. Cox, N. Magnea, K. Saminadayar, Phys. Rev. B 56, R12787 (1997). 
[32] K. Kheng, R.T. Cox, Y. Merle d'Aubigné, F. Bassani, K. Saminadayar, S. Tatarenko, Phys. Rev. Lett. 71, 1752 (1993).

[33] R.T. Cox, V. Huard, K. Kheng, S. Lovisa, R.B. Miller, K. Saminadayar, A. Arnolut, J. Cibert, S. Tatarenko, M. Potemski, Acta Phys. Pol. A 94, 99 (1998).

[34] P. Hawrylak, Phys. Rev. B 44, 3281 (1991).

[35] C.Y. Hu, W. Ossau, D.R. Yakovlev, B. König, T. Wojtowicz, G. Karczewski, J. Kossut, Acta Phys. Pol. A 94, 351 (1998).

[36] K. Kheng, K. Saminadayar, N. Magnea, J. Cryst. Growth 184/185, 849 (1998).

[37] B. Stébé, A. Ainane, Superlattices Microstruct. 5, 545 (1989).

[38] B. Stébé, G. Munschy, L. Stauffer, F. Dujardin, J. Murat, Phys. Rev. B 56, 12454 (1997).

[39] A. Keller et al., to be published.

[40] V.P. Kochereshko, D.R. Yakovlev, R.A. Suris, W. Ossau, G. Landwehr, T. Wojtowicz, M. Kutrowski, G. Karczewski, J. Kossut, J. Cryst. Growth 184/185, 826 (1998).

[41] T. Wojtowicz, M. Kutrowski, G. Karczewski, M. Potemski, F.J.T. Garcinuno, J. Kossut, to be published.

[42] R.A. Suris, D.R. Yakovlev, private information. 